\title{
BLIND CHANNEL ESTIMATION FOR CDMA SYSTEMS : AN INDUCED CYCLOSTATIONARITY APPROACH
}

\author{
W. Hachem*, F. Desbouvries*, Ph. Loubaton** \\ * Institut National des Télécommunications, 9 rue Charles Fourier, 91011 Évry, France \\ ** Université de Marne-la-Vallée, 77454 Marne-la-Vallée Cedex 2, France \\ Walid.Hachemeint-evry.fr, desboudint-evry.fr, loubaton@univ-mlv.fr
}

\begin{abstract}
In this paper, the recently introduced Transmitter Induced Cyclostationarity (TIC) techniques are applied to the CDMA uplink blind identification problem. In the proposed transmission model, the spreading code vectors are periodic, each user having a period of his own. As a consequence, a cyclic covariance function is associated to each user. This function generates a structured signal subspace which depends only on the transfer function of this user. The approach leads to clear and mild identifiability conditions. An identification algorithm is described and its theoretical asymptotic performance is presented. Simulations and comparisons with the existing stationary subspace techniques are provided. The algorithm is particularly adapted to systems where the number of users is close to the spreading factor.
\end{abstract}

\section{INTRODUCTION}

In DS-CDMA communications through a dispersive medium, accurate channel estimation prior to any detection algorithm is critical if one has to attain a high data rate. The general problem of blind channel estimation using second-order statistics of the received signal is now well understood. In the CDMA context, the spreading codes associated to the users give to the so-called signal subspace a certain structure. This fact is exploited in a number of ad hoc second-order based algorithms (see for instance [2], [5], [7], [8], [10]). In the uplink, each user transmits data over his own channel, and these algorithms estimate each channel separately. However, they work under identifiability conditions which are difficult to interpret. Furthermore, when the system is loaded, estimating each user's channel from the knowledge of the whole signal subspace and of the spreading code of that user becomes difficult.

In order to avoid these drawbacks, we propose a transmission model in which, besides the code diversity, we introduce a diversity in the "cycle domain". Our scheme can be interpreted as a multi-user generalization of the modulation precoders transmitter induced cyclostationary schemes introduced in [3] and [6]. The advantage of this method lies in the fact that we can estimate at once, and separately for each user, a signal subspace generated solely by the user's transfer function.

This paper is organized as follows. First of all, we present the stationary MIMO model which underlies the second-order subspace algorithms cited above, then we give some brief comments on these techniques. We next propose the new transmission model, for which very weak identifiability conditions are established. A theoretical analysis of the identification algorithm is performed, as well as practical simulations. They show that the method is well-suited for heavily loaded systems.

\section{PROBLEM FORMULATION}

We consider a multi-user communication system based on a direct sequence spread spectrum. Let $K$ be the number of users. User $k$ transmits a circular i.i.d. sequence $s^{k}(n)$ with variance $\sigma_{s}^{2}$. In classical systems, the spreading codes are periodic of period $L$, the spreading factor. The spreading code associated to user $k$ can thus be represented either by the constant vector $\mathbf{c}^{k}=\left[c_{0}^{k}, \ldots, c_{L-1}^{k}\right]^{T}$, or by the associated filter $c^{k}(z)=$ $\sum_{i=0}^{L-1} c_{i}^{k} z^{-i}$. For all $k$, the entries of $\mathbf{c}^{k}$ belong to the set $\{u+$ $j v, u, v \in \pm 1\}$. The overall channel transfer function associated to user $k$ (resulting from the dispersive channel, the transmit and the receive filters), sampled at chip rate, has degree $m_{k}$ and is denoted by $g^{k}(z)=\sum_{i=0}^{m_{k}} g_{i}^{k} z^{-i}$. We suppose for simplicity that $g_{0}^{k} \neq 0$ for all users, which can be guaranteed by some synchronization scheme. The received signal is sampled at chip rate. The contribution of user $k$ to this signal may be written, using operator notation, as [9] :

$$
y^{k}(n)=\left[g^{k}(z) c^{k}(z)\right] \tilde{s}^{k}(n)=\left[h^{k}(z)\right] \tilde{s}^{k}(n),
$$

where $\tilde{s}^{k}(n)$ is the zero-padded sequence $\tilde{s}^{k}(n L)=s^{k}(n)$ and $\tilde{s}^{k}(n L+l)=0$ for $l=1, \ldots, L-1$. The $L$-variate vector signal $\mathbf{y}^{k}(n)=\left[y^{k}(n L), \ldots, y^{k}(n L+L-1)\right]^{T}$ can thus be written as

$$
\mathbf{y}^{k}(n)=\left[\mathbf{h}^{k}(z)\right] s^{k}(n),
$$

in which the transfer function $\mathbf{h}^{k}(z)$ can be expressed as follows. Let $M_{k}$ be the integer satisfying $L M_{k} \leq m_{k}<L\left(M_{k}+1\right)$. Define the transfer function $\mathbf{g}^{k}(z)=\sum_{i=0}^{M_{k}} \mathbf{g}_{i}^{k} z^{-i}$, where $\mathbf{g}_{i}^{k}=$ $\left[g_{i L}^{k}, \ldots, g_{i L+L-1}^{k}\right]^{T}$ and $\mathbf{g}_{M_{k}}^{k}$ is possibly completed with zeros. Let $\mathbf{C}^{k}(z)=\mathbf{C}_{0}^{k}+\mathbf{C}_{1}^{k} z^{-1}$, where

$$
\begin{gathered}
\mathbf{C}_{0}^{k}=\left[\begin{array}{ccc}
c_{0}^{k} & \cdots & 0 \\
\vdots & \ddots & \vdots \\
c_{L-1}^{k} & \cdots & c_{0}^{k}
\end{array}\right] \\
\mathbf{C}_{1}^{k}=\left[\begin{array}{cccc}
0 & c_{L-1}^{k} & \cdots & c_{1}^{k} \\
& \ddots & \ddots & \vdots \\
\vdots & & & c_{L-1}^{k} \\
0 & \cdots & & 0
\end{array}\right]
\end{gathered}
$$


Then it can be seen that

$$
\mathbf{h}^{k}(z)=\mathbf{C}^{k}(z) \mathbf{g}^{k}(z) .
$$

The overall $L$-variate received signal is thus expressed as

$$
\mathbf{y}(n)=\sum_{k=1}^{K}\left[\mathbf{h}^{k}(z)\right] s^{k}(n)+\mathbf{w}(n)=[\mathbf{H}(z)] \mathbf{s}(n)+\mathbf{w}(n),
$$

where $\mathbf{s}(n)=\left[s^{1}(n), \ldots, s^{K}(n)\right]^{T}$, and $\mathbf{w}(n)=[w(n L), \ldots$, $w(n L+L-1)]^{T}$ is an AWGN, uncorrelated with the transmitted symbols, and with covariance $\sigma_{w}^{2} I_{L}$.

Many solutions have been proposed for solving the multiuser channel identification problem. In particular, subspace-based methods (see e.g. [2], [5], [7], [8], [10]) begin with computing the left kernel of an estimated covariance matrix, and then minimize some quadratic form. These methods do work well, provided though that $K$ is not too large. However, as we shall show, identification becomes difficult when the system gets loaded, i.e. when $K$ becomes close to $L$.

Moreover, another drawback is that the identifiability problem is not fairly well understood yet. Some sufficient identifiability conditions were proposed, but they seem difficult to interpret in terms of simple conditions on the channels and/or on the codes.

\section{IDENTIFICATION WITH TRANSMITTER INDUCED CYCLOSTATIONARITY}

In the model we propose, these drawbacks are avoided by giving the transmitted signal a cyclostationary character. To every user, we now associate a periodic spreading code vector instead of a constant one :

$$
\mathbf{c}^{k}(n)=\mathbf{c}^{k, 0}+\mathbf{c}^{k, 1} e^{2 j \pi \alpha_{k} n}
$$

where $\alpha_{k}$ is specific to user $k$. In this model, the received signal becomes

$\mathbf{y}(n)=\sum_{k=1}^{K}\left(\left[\mathbf{h}^{k, 0}(z)\right] s^{k}(n)+\left[\mathbf{h}^{k, 1}(z)\right]\left(s^{k}(n) e^{2 j \pi \alpha_{k} n}\right)\right)+\mathbf{w}(n)$

where, for $i=0,1, \mathbf{h}^{k, i}(z)=\mathbf{C}^{k, i}(z) \mathbf{g}^{k}(z), \mathbf{C}^{k, i}(z)=\mathbf{C}_{0}^{k, i}+$ $\mathbf{C}_{1}^{k, i} z^{-1}$, and the matrices $\mathbf{C}_{0}^{k, i}$ and $\mathbf{C}_{1}^{k, i}$ are associated to the code vectors $\mathbf{c}^{k, i}$ as in (3).

By construction, the signal in (7) is obviously cyclostationary with cyclic frequencies $\left\{0, \pm \alpha_{1}, \cdots \pm \alpha_{K}\right\}$ (we assume that the reader is familiar with cyclostationarity; if not, see e.g. [4]). In particular, its cyclic spectrum at cycle $\alpha_{k}$ is given by

$$
\mathbf{R}^{\alpha_{k}}(z)=\sum_{l=-M_{k}-1}^{M_{k}+1} \mathbf{R}_{l}^{\alpha_{k}} z^{-l}=\sigma_{s}^{2} \mathbf{h}^{k, 1}(z) \mathbf{h}^{k, 0 H}\left(e^{-2 j \pi \alpha_{k}} / \bar{z}\right)
$$

(where $\mathbf{h}^{k, 0^{H}}(z)$ denotes $\left(\overline{\mathbf{h}^{k, 0}(z)}\right)^{T}$ ), and thus, among all channels, depends only on the channel $\mathbf{g}^{k}(z)$ of user $k$. The channel $\mathbf{g}^{k}(z)$ can thus potentially be estimated by a single user technique from the second order cyclic statistics at cyclic frequency $\alpha_{k}$. For equation (8) to be true, note that the only requirement on the additive noise is that it has no power at the cycle $\alpha_{k}$. This requirement is trivially satisfied if, for instance, the noise is stationary.

\subsection{Identifiability}

Our purpose is to identify $g^{k}(z)$ from $\mathbf{R}^{\alpha_{k}}(z)$. Using the now classical framework of SIMO blind identification [1], we see from (8) that $\mathbf{h}^{k, 1}(z)$ is identifiable from $\mathbf{R}^{\alpha_{k}}(z)$ provided it is irreducible (i.e., $\mathbf{h}^{k, 1}(z) \neq 0$ for all $z \neq 0$, including $z=\infty$ ). Identifiability of $\mathbf{g}^{k}(z)$ follows immediately.

However, what really matters here is the identification of $\mathbf{g}^{k}(z)$, not of $\mathbf{h}^{k, 1}(z)$. Taking into account the structure of $\mathbf{h}^{k, 1}(z)$ shows that the irreducibility of $\mathbf{h}^{k, 1}(z)$ is not essential; $\mathbf{g}^{k}(z)$ is indeed identifiable under a milder sufficient condition :

Theorem 1 Let $\mathbf{g}^{k}(z)$ be irreducible. Then $\mathbf{g}^{k}(z)$ is identifiable from $\mathbf{R}^{\alpha_{k}}(z)$ up to a scalar constant.

Proof.

Let us give a constructive proof of this result. Let $\mathbf{B}(z)$ be some polynomial matrix satisfying

$$
\mathbf{B}(z) \mathbf{e}(z)=0 \Leftrightarrow \mathbf{e}(z)=\mathbf{h}^{k, 1}(z) r(z)
$$

for some scalar rational function $r(z)$. In the next subsection, we shall give a means of building such a polynomial. Now, let $\mathbf{f}(z)$ be a non zero polynomial vector, of degree lower than or equal to $M_{k}$, satisfying

$$
\mathbf{B}(z) \mathbf{C}^{k, 1}(z) \mathbf{f}(z)=0 .
$$

The transfer function $\mathbf{g}^{k}(z)$ satisfies (10), and so the set of all solutions of (10) is not empty. From (9), $\mathbf{C}^{k, 1}(z) \mathbf{f}(z)=\mathbf{h}^{k, 1}(z) r(z)$. Now, $\mathbf{C}^{k, 1}(z=\infty)=\mathbf{C}_{0}^{k, 1}$ is a regular matrix, so $\mathbf{C}^{k, 1}(z)$ is generically regular, and so $\mathbf{f}(z)=\mathbf{C}^{k, 1}(z)^{-1} \mathbf{h}^{k, 1}(z) r(z)$ coincides with $\mathbf{g}^{k}(z) r(z)$. If we assume that $\mathbf{g}^{k}(z)$ is irreducible, the equality $\mathbf{f}(z)=\mathbf{g}^{k}(z) r(z)$ implies that $r(z)$ is reduced to a polynomial. Since we assume that $\operatorname{deg}(\mathbf{f}(z)) \leq M_{k}$, necessarily $r(z)$ must reduce to a constant.

It is interesting to notice that in the case where $m_{k}<L$, i.e. if the time spread of the channel impulse response is shorter than the spreading factor, then $\mathbf{g}^{k}(z)=\mathbf{g}_{0}^{k}$ and the irreducibility condition is trivially observed : the unknown transfer function $g^{k}(z)$ is always identifiable. Though this may not be the case any longer if $m_{k} \geq L$, the irreducibility condition remains anyhow very mild. To see this, remember that $\mathbf{g}^{k}(z)$ is irreducible if and only if $g^{k}(z)$ has no $L$ zeros equidistributed on a circle centered at the origin (this result is a particular application to the SIMO case of a more general result given in [9, Proposition 2]). The property is thus expected to be generically ensured by real channels.

\subsection{TIC algorithm}

The identification procedure is split into two steps : look for one particular polynomial $\mathbf{B}(z)$ satisfying (9), then solve (10) under a non-triviality constraint on $\mathbf{f}(z)$.

Let us outline the first step, beginning with some notations. To a (polynomial or rational) $I \times J$ matrix $\mathbf{P}(z)=\sum_{i=p_{1}}^{p_{2}} \mathbf{P}_{i} z^{-i}$, we associate the generalized Sylvester matrix $\mathbf{T}_{m}(\mathbf{P})$ (of dimensions $\left.(m+1) I \times\left(m+1+p_{2}-p_{1}\right) J\right)$ defined by

$$
\mathbf{T}_{m}(\mathbf{P}) \stackrel{\text { def }}{=}\left[\begin{array}{ccccc}
\mathbf{P}_{p_{1}} & \cdots & \mathbf{P}_{p_{2}} & & 0 \\
& \ddots & & \ddots & \\
0 & & \mathbf{P}_{p_{1}} & \cdots & \mathbf{P}_{p_{2}}
\end{array}\right]
$$

For an integer $N$ greater than or equal to $M_{k}+1, \mathbf{T}_{N}\left(\mathbf{R}^{\alpha_{k}}\right)$ is the $(N+1) L \times\left(2 M_{k}+N+3\right) L$ block-Sylvester matrix associated to 
$\mathbf{R}^{\alpha_{k}}(z)$. Denote by $\boldsymbol{\Pi}$ the projection matrix on the left kernel of $\mathbf{T}_{N}\left(\mathbf{R}^{\alpha_{k}}\right)$, and partition $\boldsymbol{\Pi}$ as $\boldsymbol{\Pi}=\left[\boldsymbol{\Pi}_{0} \cdots \boldsymbol{\Pi}_{N}\right]$. Finally, denote by $\boldsymbol{\Pi}(z)$ the polynomial matrix $\boldsymbol{\Pi}(z)=\sum_{i=0}^{N} \Pi_{i} z^{-i}$. Then, it can easily be shown that $\Pi(z) \mathbf{R}^{\alpha_{k}}(z)=0$ for each $z$, and that $\Pi(z)$ satisfies (9).

As for the second step, we similarly associate to $\mathbf{P}(z)$ the generalized Sylvester matrix $\mathbf{D}_{m}(\mathbf{P})$ (of dimensions $\left(m+1+p_{2}-\right.$ $\left.\left.p_{1}\right) I \times(m+1) J\right)$ defined by

$$
\mathbf{D}_{m}(\mathbf{P}) \stackrel{\text { def }}{=}\left[\begin{array}{lll}
\mathbf{P}_{p_{1}} & & 0 \\
\vdots & \ddots & \\
\mathbf{P}_{p_{2}} & & \mathbf{P}_{p_{1}} \\
& \ddots & \vdots \\
0 & & \mathbf{P}_{p_{2}}
\end{array}\right]
$$

Let us also associate to any polynomial vector $\mathbf{f}(z)=\sum_{i=0}^{M_{k}} \mathbf{f}_{i} z^{-i}$, of dimensions $L \times 1$, the $L\left(M_{k}+1\right)$ vector $\mathbf{f}$ defined by $\mathbf{f}=\left[\mathbf{f}_{0}^{T}\right.$, $\left.\ldots, \mathbf{f}_{M_{k}}^{T}\right]^{T}$. Stated as a matrix equality, the identification equation (10) becomes

$$
\mathbf{D}_{M_{k}+1}(\boldsymbol{\Pi}) \mathbf{D}_{M_{k}}\left(\mathbf{C}^{k, 1}\right) \mathbf{f}=0
$$

In the sequel, we drop the subscripts from the notations (11) and (12) except when necessary. Equation (13) gives the means of estimating $\mathbf{g}^{k}$ up to a scalar factor : let

$$
\mathbf{Q} \stackrel{\text { def }}{=} \mathbf{D}^{H}\left(\mathbf{C}^{k, 1}\right) \mathbf{D}^{H}(\boldsymbol{\Pi}) \mathbf{D}(\mathbf{\Pi}) \mathbf{D}\left(\mathbf{C}^{k, 1}\right)
$$

Then the transfer function is estimated by solving the problem

$$
\min _{\|\mathbf{f}\|=1} \mathbf{f}^{H} \mathbf{Q} \mathbf{f}
$$

In summary, the identification algorithm consists of the following steps:

- Estimate the lags of $\mathbf{R}^{\alpha_{k}}(z)$ by

$$
\hat{\mathbf{R}}_{i}^{\alpha_{k}}=1 / T \sum_{n=0}^{T-1} \mathbf{y}(n+i) \mathbf{y}^{H}(n) e^{-2 j \pi \alpha_{k} n}
$$

where $T$ is the window size in information symbols.

- Build $\mathbf{T}\left(\hat{\mathbf{R}}^{\alpha_{k}}\right)$, then estimate $\boldsymbol{\Pi}$.

- Build $\mathbf{D}(\widehat{\Pi})$ and $\mathbf{D}\left(\mathbf{C}^{k, 1}\right)$, then solve (15).

\section{ASYMPTOTIC STATISTICAL ANALYSIS}

In this section, it is assumed without loss of generality that the true vector $\mathbf{g}^{k}=\left[\left(\mathbf{g}_{0}^{k}\right)^{T}, \ldots,\left(\mathbf{g}_{M_{k}}^{k}\right)^{T}\right]^{T}$ satisfies $\left\|\mathbf{g}^{k}\right\|^{2}=\sum_{i=0}^{m_{k}}\left|g_{i}^{k}\right|^{2}$ $=1$. Under this assumption, minimizing the quadratic form (15) gives an estimate of $\mathbf{g}^{k}$, up to a phase ambiguity $e^{j \phi}$. It is known that this ambiguity cannot be raised by the sole use of second order methods. In our performance calculations, we implicitely choose the determination of the estimate $\hat{\mathbf{g}}^{k}$ that satisfies $\left(\hat{\mathbf{g}}^{k}\right)^{H} \mathbf{g}^{k} \geq 0$, thus raising this ambiguity. We give the following performance result, the proof of which we skip for want of space.

Theorem 2 Let the transmission model be described by (7), where the i.i.d. symbols $s^{k}(n)$ are complex and circular (i.e $\forall \tau, E\left(s^{k}(n+\right.$ $\left.\tau) s^{k}(n)\right)=0$ ), and the noise $\mathbf{w}(n)$ is stationary, Gaussian and uncorrelated with the symbols. Suppose that the FIR transfer function $\mathbf{g}^{k}(z)$ associated to user $k$ is irreducible, and that its degree $M_{k}$ is known. Let $\hat{\mathbf{g}}^{k}$ be the estimate given by the algorithm described above. Then the asymptotic covariance of the stochastic process $\sqrt{T}\left(\hat{\mathbf{g}}^{k}-\mathbf{g}^{k}\right)$ is given by

$$
\Sigma_{\mathbf{g}}=\mathbf{Q}^{\#} \mathbf{D}^{H}\left(\mathbf{C}^{k, 1}\right) \mathbf{D}^{H}(\boldsymbol{\Pi})\left(\Sigma_{\mathcal{N}}+\Sigma_{\kappa}\right) \mathbf{D}(\boldsymbol{\Pi}) \mathbf{D}\left(\mathbf{C}^{k, 1}\right) \mathbf{Q}^{\#},
$$

where $\mathbf{Q}$ is given by (14) and

$$
\begin{aligned}
& \left.\Sigma_{\mathcal{N}}=\frac{1}{2 \pi} \sum_{\beta} \frac{e^{-2 j \pi\left(M_{k}+1\right) \beta} \int_{0}^{2 \pi}\left(\mathbf{T}_{N}^{T}\left(\mathbf{h}^{k, 1}\right) \boldsymbol{\Gamma}^{T}\left(e^{-j \lambda}\right)\right.}{\left.\mathbf{R}^{\beta}\left(e^{j\left(\lambda-2 \pi \alpha_{k}\right)}\right) \boldsymbol{\Gamma}\left(e^{-j(\lambda-2 \pi \beta}\right)\right) \mathbf{T}_{N}\left(\mathbf{h}^{k, 1}\right)}\right) \\
& \left(\boldsymbol{\Pi}\left(e^{j \lambda}\right) \mathbf{R}^{\beta}\left(e^{j \lambda}\right) \boldsymbol{\Pi}^{H}\left(e^{j(\lambda-2 \pi \beta)}\right)\right) d \lambda, \\
& \Sigma_{\kappa}=-\frac{\sigma_{s}^{4}}{4 \pi^{2}} \sum_{l=1}^{K} \sum_{u-v=p-q} \mathbf{w}(l, u, v) \mathbf{w}(l, p, q)^{H}, \\
& \boldsymbol{\Gamma}=\mathbf{T}\left(\mathbf{R}^{\alpha_{k}}\right)^{H}\left(\mathbf{T}\left(\mathbf{R}^{\alpha_{k}}\right) \mathbf{T}\left(\mathbf{R}^{\alpha_{k}}\right)^{H}\right)^{\#} \\
& =\left[\boldsymbol{\Gamma}^{T}(0) \ldots \boldsymbol{\Gamma}^{T}\left(2 M_{k}+2+N\right)\right]^{T} \text {, } \\
& \boldsymbol{\Gamma}\left(e^{j \lambda}\right)=\sum_{l=0}^{2 M_{k}+2+N} \boldsymbol{\Gamma}(l) e^{-j l \lambda}, \text { and } \\
& \mathbf{w}(l, u, v)=\int_{0}^{2 \pi}\left(\mathbf{T}_{N}^{T}\left(\mathbf{h}^{k, 1}\right) \boldsymbol{\Gamma}^{T}\left(e^{-j \lambda}\right) \overline{\mathbf{h}^{l, v}\left(e^{j\left(\lambda-2 \pi \alpha_{k}\right)}\right)}\right) \\
& \left(\boldsymbol{\Pi}\left(e^{j \lambda}\right) \mathbf{h}^{l, u}\left(e^{j \lambda}\right)\right) e^{-j\left(M_{k}+1\right) \lambda} d \lambda .
\end{aligned}
$$

\section{SIMULATIONS}

In our computer experiments, we simulated a CDMA uplink transmission across a multipath microwave channel. The transmitted symbols $\left\{s^{k}(n)\right\}$ belong to a QPSK constellation. A perfect power control ensures that all users transmit at equal power. In the first two figures, the mean square error of the channel estimates $E\left(\left\|\hat{\mathbf{g}}^{k}-\mathbf{g}^{k}\right\|^{2}\right)$ is displayed and our algorithm is compared with a stationary subspace algorithm. In these figures, the MSE is averaged over the users, and over different realizations of the channels (since amplitudes and delays of radio pathes are stochastic) and of the transmitted sequences. The signal to noise ratio is $15 \mathrm{~dB}$. The dashed curves are obtained by Monte-Carlo simulations while the plain curves illustrate the theoretical results. For the TIC algorith$\mathrm{m}$, these are predicted by (18), while for the subspace algorithm, they can be derived by similar techniques.

Figures 1 and 2 differ only by the number $K$ of users. In figure 1, we chose $L=8$ and $K=4$. Both simulations and theory show that the subspace algorithm outperforms the TIC algorithm. As a matter of fact, the signal subspace is estimated with far better accuracy in the stationary transmission scheme. In the absence of additive noise, this subspace is estimated exactly. As for the TIC algorithm, the estimation of $\mathbf{R}^{\alpha_{k}}(z)$ is corrupted by the signals of the other users. This effect clearly dominates that of additive noise.

In the stationary context, the exact quadratic form used to estimate the channel is usually well-conditioned when there are few users. This is no longer the case if their number becomes close to 
the spreading factor. In figure 2, the conditions are the same as in figure 1 except for the number of users, which is now equal to 7 . It is clear that the stationary algorithm cannot be used in this case.

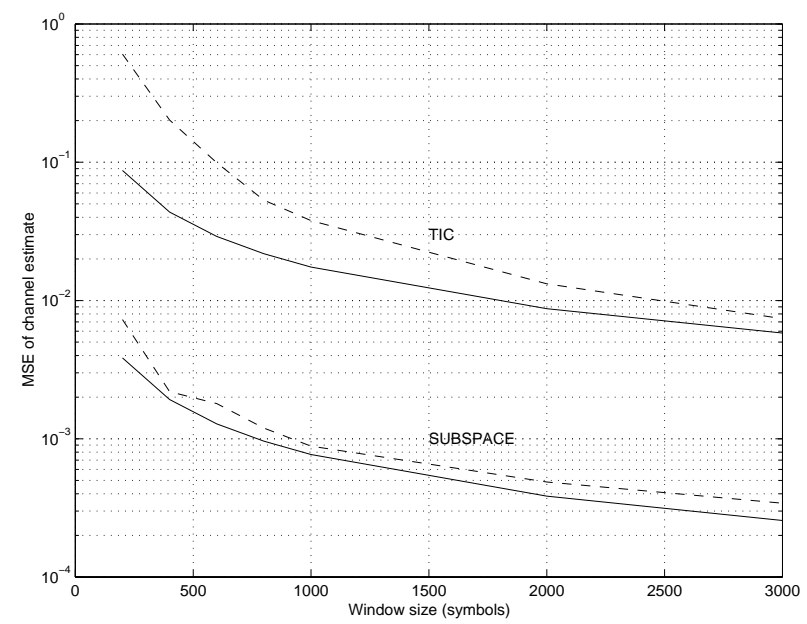

Figure 1: Channel estimation, $L=8, K=4$

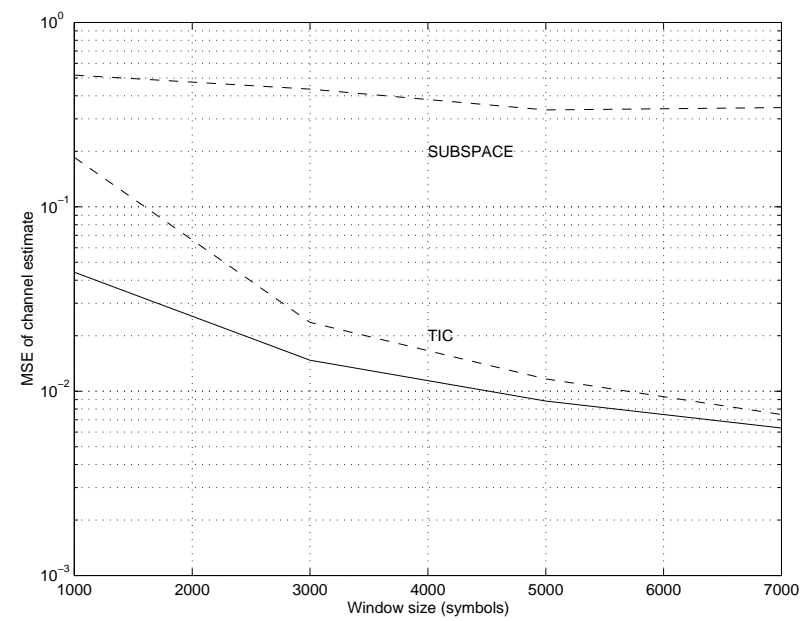

Figure 2: Channel estimation, $L=8, K=7$

Lastly, figure 3 shows the performance of the TIC algorithm in terms of bit error rate. Conditions are the same as for figure 2. After estimating all the contributing channels, a multiuser detection algorithm is applied on the received signal. The main device in the receiver is a $L$-input $K$-output polyperiodic Wiener filter (see [4]) deduced from the estimated channels. The bit error rate (BER) is averaged over the users, and over different realizations of the channels and of the transmitted sequences. In fact, simulation$\mathrm{s}$ show that for the values of the channel estimation window size in symbols considered here, the BER is more sensitive to channel misestimation rather than to the additive noise. This is the reason why BER is diplayed versus $T$. For this heavily loaded system, the BER is approximately equal to $10^{-2}$ for a channel estimation window size of 3500 symbols.

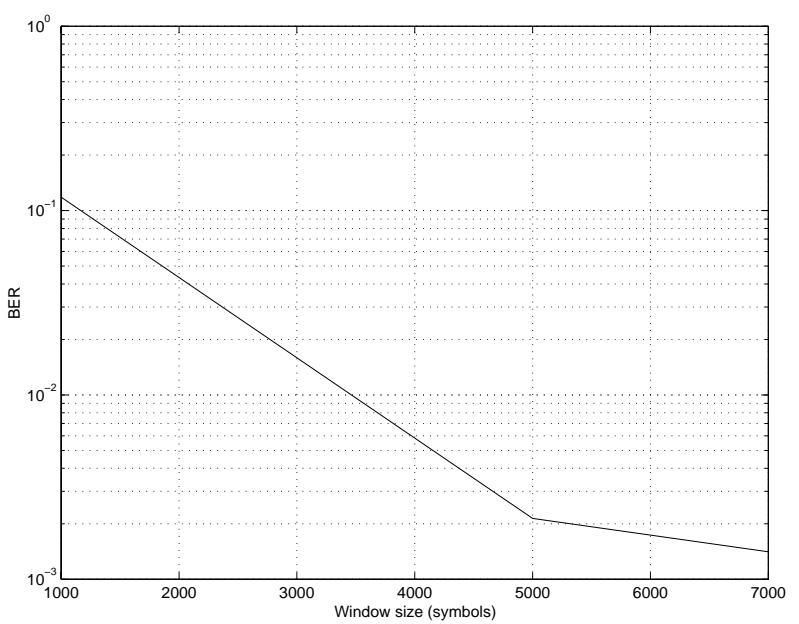

Figure 3: Bit error rate, $L=8, K=7$

\section{REFERENCES}

[1] K. Abed-Meraim, P. Loubaton and E. Moulines, "A Subspace Algorithm for Certain Blind Identification Problems", IEEE Transactions on Information Theory, Vol. 43, No. 2, March 1997

[2] S. Bensley and B. Aazhang, "Subspace-Based Channel Estimation for Code Division Multiple Access Communication Systems", IEEE Transactions on Communications, Vol. 44, No. 8, August 1996

[3] A. Chevreuil, P. Loubaton, "Blind Second Order Identification of FIR Channels: Forced Cyclostationarity and Structured Subspace Method", IEEE Signal Processing Letters, July 1997

[4] "Cyclostationarity in Communications and Signal Processing", W. A. Gardner Ed., IEEE Press, 1994

[5] H. Liu and G. Xu, "A Subspace Method for Signature Waveform Estimation in Synchronous CDMA Systems," IEEE Transactions on Communications, Vol. 44, No. 10, October 1996

[6] E. Serpedin, G.B. Giannakis, ”Blind Channel Identification and Equalization with Modulation Induced Cyclostationnarity", IEEE Transactions on Signal Processing, Vol. 46, No. 7, July 1998

[7] M. Torlak and G. Xu, "Blind Multiuser Channel Estimation in Asynchronous CDMA Systems," IEEE Transactions on Signal Processing, Vol. 45, No. 1, January 1997

[8] M. Tsatsanis and G. Giannakis, "Multirate Filter Banks for Code-Division Multiple Access Systems," Proceedings of the ICASSP-95, Detroit, 1995, Vol. 2

[9] M. Tsatsanis and G. Giannakis, "Optimal Decorrelating Receivers for DS-CDMA Systems: A Signal Processing Framework", IEEE Transactions on Signal Processing, Vol. 44, No. 12, December 1996

[10] X. Wang and H.V. Poor, "Blind Equalization and Multiuser Detection in Dispersive CDMA Channels", IEEE Transactions on Communications, Vol. 46, No. 1, January 1998 